\title{
Using Social Networks in Personnel Selection: A Survey of Human Resource Professionals
}

\author{
Nuno Melão \\ CISeD - Research Centre in Digital Services \\ Polytechnic of Viseu, Portugal \\ Viseu, Portugal \\ nmelao@estgv.ipv.pt
}

\author{
João Reis \\ Department of Economics, Management, Industrial \\ Engineering and Tourism and GOVCOPP \\ University of Aveiro \\ Aveiro, Portugal \\ reis.joao@ua.pt
}

\begin{abstract}
Although the analysis of social media to aid decision making in Human Resource (HR) selection is becoming commonplace, there is little empirical research on this phenomenon. This study reports on the use of Social Networking Sites (SNS) by HR professionals to assist applicant selection. It employs a survey methodology based on a cross-sectional questionnaire which was administered to a sample of 10000 professionals in Portugal. The response rate was $10.5 \%$ and the results show that $41 \%$ of the respondents use SNS in employee selection. The most used SNS are LinkedIn (89\%) and Facebook $(58 \%)$, and the evaluation of applicant profiles usually takes place before the first interview. Around three quarters of participants indicate to assess applicants by forming a general impression of their adjustment to the organization. The most regularly inferred constructs from profiles are work experience, education and training, written communication skills, maturity and professionalism. Respondents infer more often the outcome person-organization adjustment. The conclusion is that for many Portuguese organizations SNS seem to be a useful complementary tool to the most conventional personnel selection techniques.
\end{abstract}

Keywords - Social Networking Websites; personnel selection; cybervetting; human resource management; practice.

\section{INTRODUCTION}

Personnel selection is a key process in organizations given that it is directly responsible for the identification and acquisition of applicants with high levels of knowledge and skills that can contribute to organizational performance [1]. As Social Networking Sites (SNS) have become widespread, so too has the availability of information about applicants both from work and non-work domains. This, in turn, has led many organizations to incorporate this new source of information into their selection processes. The popular press [2], practitioner $[3,4]$ and scholar literature $[5,6,7]$ confirm that this practice is becoming widespread. However, the use of this source of information in personnel selection is replete with dangers, including those associated with violations of privacy and laws, discrimination, lack of reliability and validity $[8,9$, $10,11,12,13]$.

Although the investigation of the aforementioned issues is an important research avenue, it is also essential to examine current practice about the use of SNS in personnel selection as this enables a better understanding of its merits and impacts to individuals and organizations alike. Surprisingly, not many scholarly studies exist that explore how Human Resource (HR) professionals employ SNS in selection processes. Noteworthy examples in this respect are Berkelaar [14], Caers and Castelyns [15], Henderson [16], and Hoek et al. [17]. Furthermore, the majority of such studies were performed in the USA, and several authors [14, 17] have highlighted the need to explore this phenomenon in other countries so as to enhance our understanding of existing practice.

This paper explores the use of SNS in personnel selection through a survey of 1052 HR professionals in Portugal. The structure of the paper is as follows. First, it reviews the relevant empirical literature on this emerging field. Then, after explaining the methodological choices and procedures, it presents and discusses the findings of the survey. Finally, it draws conclusions and some implications for research and practice.

\section{LITERATURE REVIEW}

It is increasingly common for HR professionals to analyse profiles from SNS to select job applicants [18]. For example, CareerBuilder and the Society for Human Resource Management (SHRM) conduct regularly large-scale industry surveys in the USA, which have been consistently confirming this practice. A CareerBuilder [4] survey reports that $70 \%$ of private sector organizations use social media to screen applicants, an increase of $10 \%$ when compared to 2016. Of those who use SNS, $61 \%$ search for information that supports their qualifications for the job, $50 \%$ check if the applicant has a professional online persona, $37 \%$ search for what others are posting about the applicants, and $24 \%$ search for a reason not to hire an applicant. Furthermore, 54\% disqualified an applicant due to concerning content found in SNS profiles. The SHRM [3] surveyed in 2015 its members, who typically have specific HR management education and experience, finding that $43 \%$ use social media or online search engines to screen applicants. The reasons for this are, among others, to obtain more information about an applicant (61\%), to verify information from the curriculum vitae $(\mathrm{CV})$, résumé and cover letter (50\%), and because applicants include links to their SNS profiles on résumés $(41 \%)$. Within the HR selection process, LinkedIn and Facebook are the most used SNS; $36 \%$ reply that 
their use varies by job level, $28 \%$ use them before the interview, $20 \%$ use them after the interview but before a job offer, and $9 \%$ use them before a contingent job offer.

Although many practitioners have been using SNS for employee selection, scholars have yet to fully investigate this phenomenon. Indeed, not many empirical studies have been conducted to date to ascertain current practice. One of the earliest is Caers and Castelyns' [15] survey study of Belgian HR professionals, having found that a large proportion of participants reported to use LinkedIn and (to a lesser extent) Facebook to find additional information about applicants; some participants also reported to use LinkedIn and Facebook to decide whether an applicant should be invited to an interview. Interestingly, around $40 \%$ of respondents believed that the profile picture gives cues about extraversion and maturity. The authors go on to conclude that by using the profile content to make inferences about the personality and behavior of the applicant, selectors may add biases in the initial steps of the selection process.

An additional contribution worth mentioning is Hoek et al. [17], who describe the results of 15 qualitative interviews with HR professionals from New Zealand with the aim to learn how they use SNS to select applicants. Respondents stated to gather interview cues when checking profiles before an interview, and stated to validate information transmitted by the applicant when examining profiles after an interview. With regard to candidate fit, the authors found that LinkedIn served to evaluate job fit and hard skills, whereas Facebook assisted to determine organization fit and soft skills. However, they added that participants were mainly interested in getting "a rounded understanding" of the applicant, i.e. assessing personorganization fit. Interestingly, most of the reasons mentioned for not using SNS in personnel selection seemed to be associated with resource issues. Overall, they found evidence that SNS are not used necessarily as a selection method per se, but rather as a complementary tool.

In one of the most comprehensive qualitative studies on this topic, Berkelaar [14] reported on the results of an interview research in the USA conducted to ascertain how the use of SNS is changing existing personnel selection practices. Participants mentioned that the general purpose was to evaluate the "overall person to a get sense of fit", but also unveiled three specific purposes: to drop applicants with problematic behaviour (e.g. lack of relational skills) or "red flags" (e.g. gaps in work history); to automate the selection process (e.g. collecting applications or interacting with applicants), making it more efficient; and to speed up and develop a deeper understanding of applicants. Consistent with the findings of Hoek et al. [11], most participants mentioned using SNS as a supplement to conventional personnel selection practices.

A recent study is that of Henderson [16] who conducted a survey of $195 \mathrm{HR}$ professionals in the USA to examine four issues, namely, the reasons for reviewing SNS profiles, constructs that practitioners are attempting to infer, reasons that lead practitioners to screen out applicants, and the reasons for not using SNS in personnel selection. This study confirmed previous results in that LinkedIn and Facebook are the most used SNS in personnel selection, along with that most professionals review profiles before the first interview. The findings of this research painted a slightly different picture then previous studies. In addition to wishing to obtain an overall impression of applicants, participants stated to check profiles because they wanted to assess the applicants' professionalism, they had curiosity or were available, and they sought to check the applicants' online persona. The majority of the respondents reported to infer professionalism, while constructs such as work experience, communication skills, discrepancies, and interests accounted for a small percentage each. When asked why they rejected applicants, almost all respondents mentioned that it was because they found inappropriate behaviour in profiles (e.g. inappropriate posts). In contrast with Hoek et al. [17], the vast majority of reasons for not using SNS were not concerned with resource issues, but with the presence of extraneous information and legal concerns. Compared with previous research, the findings of this study gave further stress to professionalism.

Overall, these studies have provided useful information toward understanding the use of SNS by HR professionals in personnel selection. Nevertheless, as Henderson [16] pointed out "it is alarming how little we know about how social media is being used during the selection process" (p. 1). The majority of the aforementioned empirical works have been carried out in the USA, and, specifically, Berkelaar [14] and Hoek et al. [17] have mentioned the value of exploring current practice with larger samples and in different countries. Doing this would enable a more thorough comprehension of the phenomenon along with its worth and impacts for organizations and applicants.

\section{METHODOLOGY}

We address the following research question:

\section{RQ) How HR professionals report using SNS in personnel selection?}

This work employs a cross-sectional, questionnaire-based survey methodology. The sample is composed of 10000 organizations which were randomly selected from a population of 28007 active organizations in Portugal, with a minimum of 10 employees and an email address, listed in the SABIC database, at the date of July $7^{\text {th }}, 2017$.

We started by conducting a literature review to assist designing the questionnaire. A few questions were adapted from the SHRM's survey [3] for validation purposes; we also included some open-ended questions to obtain further clarifications from respondents. After completing the first draft, we asked five academics and five HR professionals to check the consistency and clearness of the instrument, and made a few changes accordingly. The final instrument contained six sections: 1) organization/participant profile; 2) use of SNS; elements of profile analyzed; 3 ) knowledge, skills and abilities assessed; 4) assessment outcomes; and 5) other comments. A five-point Likert scale (1=never, 5=always) was used in sections 3 to 5 . A copy of the questionnaire may be obtained from the first author upon request. The results of section 3 will not be reported here, as they are part of a different research project. 
The questionnaire was administered during July by sending an email addressed to the director of HR department inviting her or other person who might be in a better position to participate in the study. To encourage participants to complete the survey, we gave confidentiality assurances, offered a report summarizing the findings, and a prize draw. A reminder was sent after approximately 15 days.

A total of 1052 complete responses were obtained, which corresponds to a response rate of $10.5 \%$. Table 1 displays the sample profile. The majority of respondents are female $(65 \%)$, while $35 \%$ are males. Most of them have a bachelor's degree $(60 \%)$ or a master/doctorate degree $(22 \%) .38 \%$ have between 11 and 20 years of experience, and only $7 \%$ have less than three years of experience. More than $50 \%$ are HR managers (54\%), whereas $18 \%$ are applicant's supervisors. In regard to the type of organization, small organizations represent $53 \%$, medium-sized organizations $29 \%$, and large organizations $18 \%$. $34 \%$ of the respondents work in service sector organizations, $26 \%$ in manufacturing organizations, and $19 \%$ in wholesale and retail trade organizations.

TABLE I. PROFILE OF PARTICIPANTS (N=1 052)

\begin{tabular}{|c|c|c|}
\hline & n & $\%$ \\
\hline \multicolumn{3}{|l|}{ Gender } \\
\hline Female & 688 & $65 \%$ \\
\hline Male & 364 & $34 \%$ \\
\hline \multicolumn{3}{|l|}{ Education } \\
\hline Master's or doctorate degree & 236 & $22 \%$ \\
\hline Bachelor's degree & 626 & $60 \%$ \\
\hline Other & 190 & $18 \%$ \\
\hline \multicolumn{3}{|l|}{ Professional experience } \\
\hline Under 3 years & 75 & $7 \%$ \\
\hline $3-5$ & 103 & $10 \%$ \\
\hline $6-10$ & 172 & $16 \%$ \\
\hline $11-20$ & 399 & $38 \%$ \\
\hline 21 or more & 303 & $29 \%$ \\
\hline \multicolumn{3}{|l|}{ Role } \\
\hline HR assistant & 290 & $28 \%$ \\
\hline HR manager & 571 & $54 \%$ \\
\hline Applicant's supervisor & 191 & $18 \%$ \\
\hline \multicolumn{3}{|l|}{ Number of employees } \\
\hline Under 50 (small) & 560 & $53 \%$ \\
\hline 50-249 (medium-sized) & 301 & $29 \%$ \\
\hline 250 or more (large) & 191 & $18 \%$ \\
\hline \multicolumn{3}{|l|}{ Activity sector } \\
\hline Agriculture, forestry and fishing & 31 & $3 \%$ \\
\hline Mining & 5 & $1 \%$ \\
\hline Construction & 81 & $8 \%$ \\
\hline Manufacturing & 277 & $26 \%$ \\
\hline $\begin{array}{l}\text { Transportation, communications, } \\
\text { electric, gas and sanitary service }\end{array}$ & 61 & $6 \%$ \\
\hline Wholesale and retail trade & 200 & $19 \%$ \\
\hline Finance, insurance and real estate & 20 & $2 \%$ \\
\hline Services & 353 & $34 \%$ \\
\hline Public administration & 24 & $2 \%$ \\
\hline
\end{tabular}

\section{RESULTS AND DisCUSSION}

More than half of respondents (59\%) claimed not using SNS in personnel selection. The most commonly cited reasons for non-use were that the information provided was poorly/not related to job performance $(52 \%)$, potential privacy issues (30\%), and difficulty in standardizing assessments among all applicants (26\%). On the other hand, the unfavorable cost/benefit ratio (18\%), potential legal and ethical problems $(16 \%)$, and potential negative reaction of applicants $(8 \%)$ were the less mentioned reasons. Table 2 presents the results for the reasons of non-use. Compared with the surveys of SHRM [3] and Henderson [16], the reason potential legal and ethical issues received less emphasis, suggesting that participants perceived this as less of an obstacle.

TABLE II. REASONS FOR NON-USE $(\mathrm{N}=623)$

\begin{tabular}{|l|c|c|}
\hline \multicolumn{1}{|c|}{ Reasons } & $\mathbf{n}$ & $\mathbf{\%}$ \\
\hline Potential legal and ethical issues & 99 & $16 \%$ \\
\hline Potential privacy issues & 184 & $30 \%$ \\
\hline $\begin{array}{l}\text { Information provided is little or not related to } \\
\text { job performance }\end{array}$ & 322 & $52 \%$ \\
\hline $\begin{array}{l}\text { Difficulty to standardize assessments across } \\
\text { all applicants }\end{array}$ & 159 & $26 \%$ \\
\hline $\begin{array}{l}\text { The cost of time required to assess applicants } \\
\text { outweighs the benefit of added information }\end{array}$ & 114 & $18 \%$ \\
\hline Potential negative reaction from applicants & 47 & $8 \%$ \\
\hline Other & 123 & $20 \%$ \\
\hline
\end{tabular}

Around $41 \%$ of respondents mentioned to use SNS in personnel selection. This figure compares against $43 \%$ and $70 \%$ of, respectively, the 2015 SHRM's [3] and 2017 CareerBuilder's [4] surveys in USA. Respondents replied that this use was mainly because SNS complement the information from the CV and cover letter $(65 \%)$, are a fast and efficient method (63\%), enable to assess whether applicants would be a good fit to the organization (34\%), and enable to check the information provided by the applicant's CV and cover letter (32\%). Other less cited reasons include they enable to draw inferences about the applicant's future job performance (11\%), and they avoid negligent hiring issues (4\%). Table 3 shows the results of the usage reasons question. In contrast with SHRM's survey [3], the possibility of verifying information received a lower proportion of replies, which may indicate that that SNS are less used here as a type of background check. While the reasons uncovered are diverse, this study reinforces the complementary role of SNS in selection.

TABLE III. REASONS FOR USING SNS (N=429)

\begin{tabular}{|l|c|c|}
\hline \multicolumn{1}{|c|}{ Reasons } & n & $\%$ \\
\hline Quick and efficient method & 270 & $63 \%$ \\
\hline Avoid negligent hiring issues & 17 & $4 \%$ \\
\hline $\begin{array}{l}\text { Complement the information provided by } \\
\text { applicant's CV and cover letter }\end{array}$ & 279 & $65 \%$ \\
\hline $\begin{array}{l}\text { Enable to check the information provided by } \\
\text { applicant's CV and cover letter }\end{array}$ & 136 & $32 \%$ \\
\hline $\begin{array}{l}\text { Enable to draw inferences about applicants' } \\
\text { future job performance }\end{array}$ & 49 & $11 \%$ \\
\hline $\begin{array}{l}\text { Enable to assess whether applicants would be a } \\
\text { good fit to the organization }\end{array}$ & 144 & $34 \%$ \\
\hline Other & 31 & $7 \%$ \\
\hline
\end{tabular}

When asked about which SNS are used in personnel selection (Fig. 1), the vast majority of selectors replied LinkedIn $(89 \%)$ and Facebook $(58 \%)$. These were followed by social networks focused on certain professions (e.g., GitHub and Stack Overflow for computer programmers) or on certain countries (e.g., Xing focuses on the German-speaking countries) (24\%), while Google+, Twitter, and Instagram obtained a very low number of replies. Fig. 1 depicts the SNS 
reported to be used in personnel selection. These results are largely in harmony with those of other recent surveys, such as [3] and [16], though Twitter received a much higher proportion of use in these two latter surveys.

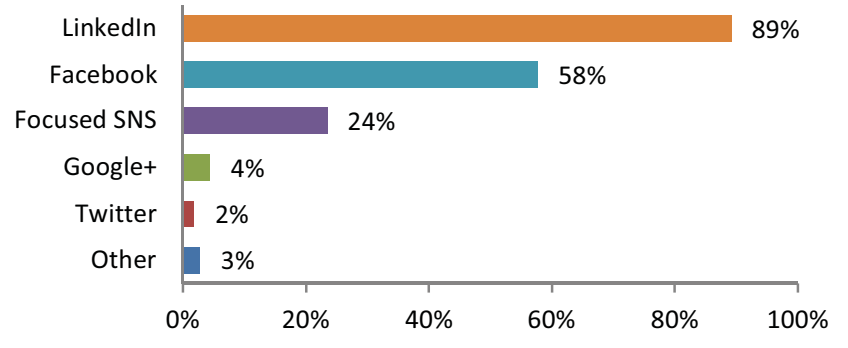

Figure 1. Types of SNS used $(n=429)$.

The questionnaire next asked about the use of SNS across the selection process (Fig. 2). Most selectors indicated to use SNS before interviewing applicants for all types of positions ( $76 \%$ for management positions vs $64 \%$ for other positions). Contrarily, respondents reported a far inferior usage in the later stages of the selection process $-17 \%$ after the interviews but before a job offer for management positions (against 20\% for other positions), and $2 \%$ after a conditional job offer for any type of positions. These findings are analogous to those of other studies $[3,16]$, providing further evidence that SNS are mainly used in the initial stages of the selection process.

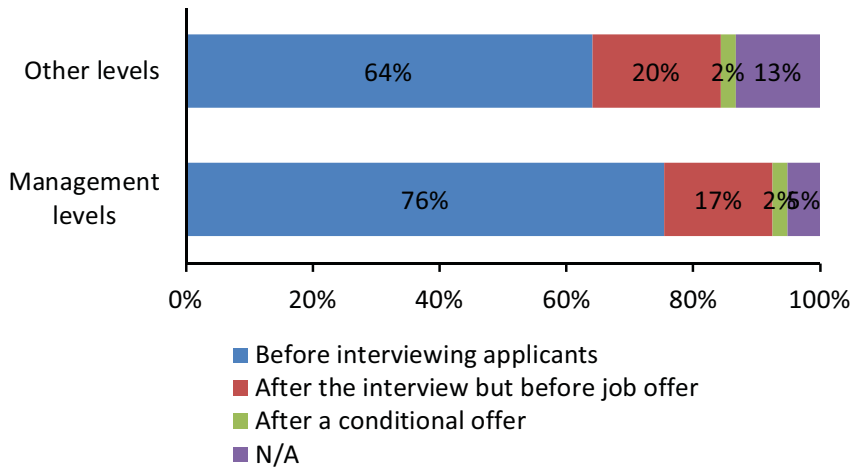

Another question asked how participants assess SNS profiles (see Fig. 3). Around 75\% indicated to make an overall impression about the applicant's fit to the organization. This accords with Berkelaar [14] and Hoek et al. [17], whose studies suggest that selectors are concerned with obtaining an overall understanding of the applicant. A small proportion (12\%) declared to evaluate applicants by disqualifying them when problematic content is found. This contradicts Roth et al.'s [6] observation that SNS are primarily employed to pursue that objective. The evaluation of profiles through a scale or protocol was reported by just $10 \%$, while $2 \%$ reported using other methods. It is important to note that this is the first time that the use of scales or protocols is reported in scholarly empirical studies.

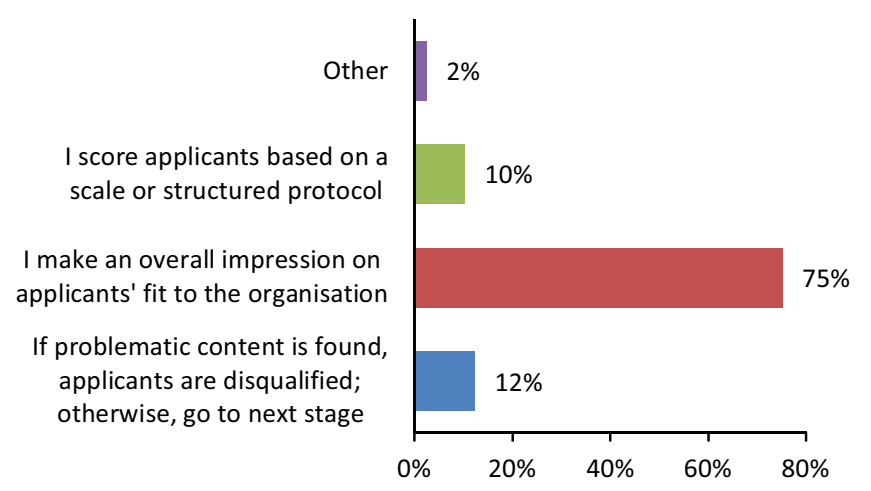

Figure 3. Approaches used to assess SNS profiles $(n=429)$.

The next question inquired what knowledge, skills and abilities (KSA) are being inferred when profiles are being analyzed (see Fig. 4). The most frequently inferred are work experience $(M=4.2)$, education and training $(M=4.1)$, written communication skills $(\mathrm{M}=3.8)$, maturity and professionalism $(M=3.6)$, interests and preferences $(M=3.6)$, and interpersonal skills $(M=3.5)$. Physical attributes $(M=1.9)$, cognitive abilities $(\mathrm{M}=2.9)$, and persuasion and negotiation skills $(\mathrm{M}=3)$ are less frequently inferred. Personality $(M=3.4)$, creativity $(M=3.4)$, dependability (3.4), moral values and standards $(\mathrm{M}=3.3)$, and leadership skills $(\mathrm{M}=3.2)$ are inferred with moderate regularity.

Figure 2. Use of SNS by professional levels $(n=429)$.

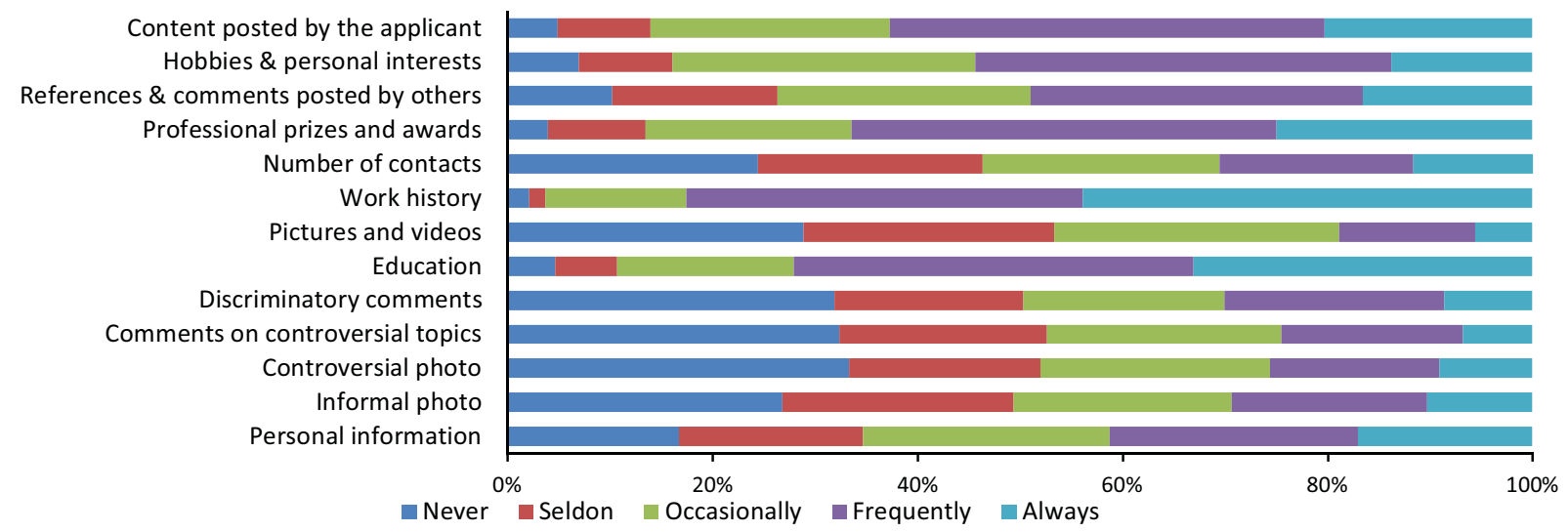

Figure 4. Knowledge, skills, and abilities being inferred $(n=429)$. 
Overall, these results are in clear contrast with those of Henderson's [16], who found that participants inferred mainly professionalism, whereas only $13 \%$ looked at work experience.

Additionally, we asked participants about the outcomes they were inferring when they are assessing profiles (Fig. 5). The most inferred was person-organization fit $(M=3.6)$. This result is consistent with what has been reported in Hoek et al. [17] in that selectors wish to obtain a rounded understanding of applicants, i.e. person-organization fit. Despite being the most inferred outcome, its average value is rather low, which suggests that some participants are solely attempting to infer $\mathrm{KSA}$ from profiles rather than inferring outcomes. Another inferred outcome was job-fit $(\mathrm{M}=2.9)$. Absenteeism and turnover were rarely inferred $(\mathrm{M}=2.5, \mathrm{M}=2.3)$.

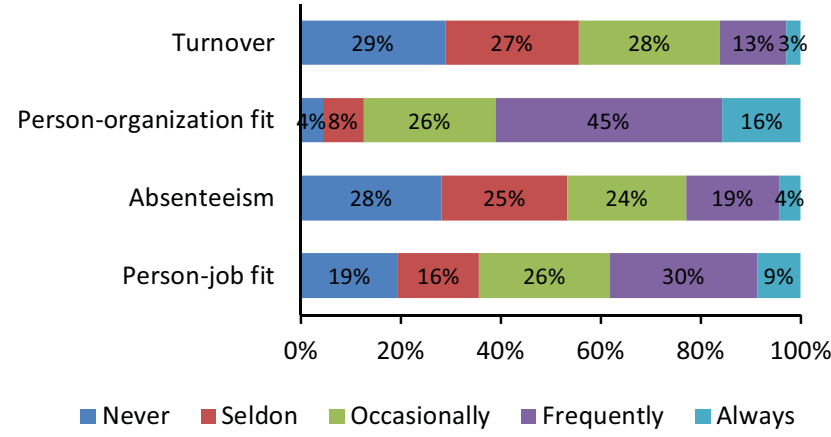

Figure 5. Outcomes being inferred $(n=429)$.

The last question sought feedback from participants on other general comments, with a total of 25 answers. Below is a selection of comments.

- "If the candidate matches the desired profile, the interview will always be the last phase of the process and social networks can help to conduct this interview."

- "All the profile revealed on LinkedIn is valid but as a complement to the CV and interview; I usually take the printed profile to the interview."

- “The information on the applicants' profiles, especially on professional networks like LinkedIn, is a good complement to the selection process, which should be used more in the future as an extra source of information."

- "The information contained in social networks has to be evaluated carefully as it is little reliable (people put untrue or inaccurate facts there)."

- "The information from social networks, although public and/or published, should not be a relevant basis for getting to know the applicants. The social roles played by individuals, when removed from their context of action, may induce biased interpretations."

The first three comments highlight the complementary role of SNS in the selection process. The other comments make two cautionary notes. The first points out that profiles may not reflect actual personas because applicants may wish to create a positive impression on the hiring professional. This issue also emerged in the study of Hoek et al. [17]. The second refers to the dangers of using profile content, specifically that extracted from SNS with a high context collapse (such as Facebook), to make inferences about applicants, as it may not be relevant or may be ill suited. While investigating this issue, Pike et al. [12] concluded that SNS with a high context collapse may actually add ambiguity in the selection process.

\section{CONCLUSION}

This paper reports on the use of SNS in personnel selection through a survey of HR professionals. To our best knowledge, this is the first empirical study of its kind to be conducted in Portugal. The prevalence of use is lower in Portugal than in more developed countries like the USA. Considering that smaller organizations tend to have less hiring needs and that more than $99 \%$ of Portuguese organizations are of small and medium size, this result may not come as a surprise. Results indicated that participants use SNS mainly due to reasons of complementarity, efficiency, fit assessment, and validity. This, in turn, begs the attention of researchers to investigate whether resource-based theory, transaction cost theory, or information systems adoption theories help explain why SNS is used in personnel selection.

This study provides further evidence that profile analysis in mainly conducted during the initial stages of the selection process (i.e., before the face-to-face interview) as well as that such analysis tends to be performed in an intuitive, nonsystematized way by formulating an overall impression of the applicant's fit to the organization. What is less clear, though, is how SNS are used within the different stages of the selection process, and thus this seems a research avenue worth pursuing further. An interesting insight that emerged from the survey is that practitioners are using, albeit in a small percentage, scales or protocols to assess profiles. While this has the potential to improve the reliability of assessments, the effectiveness of this approach depends on the way they are employed. An implication for scholars is that further in-depth research needs to be conducted so as to ascertain what type of scales are using as well as how do practitioners use them to make assessments. Furthermore, while formulating an overall impression, practitioners attempt to infer various KSA about the applicant. However, we still know very little about the pieces of information, decision processes and heuristics that practitioners use to make such inferences, which constitutes another path for further research.

This work is not without limitations. Despite the large sample, this study focused only on Portugal, and, therefore, the results reported here might be not transferable to other countries. Future studies could look at ways to extend this work to an international level. The methodology used herein is not suitable to generate in-depth knowledge on the dynamics and processes of use of SNS in personnel selection. In-depth interviews or case studies could assist scholars in going beyond what has been possible in this research.

\section{ACKNOWLEDGMENT}


This work is funded by National Funds through the FCT Foundation for Science and Technology, I.P., within the scope of the project Ref. UIDB/05583/2020. We would like to thank the Research Centre in Digital Services (CISeD) and the Polytechnic of Viseu for their support.

\section{REFERENCES}

[1] Y. Kim, and R. Ployhart, "The strategic value of selection practices: antecedents and consequences of firm-level selection practice usage," Academy of Management Journal, vol. 61, n. 1, pp. 46-66, 2018.

[2] L. Quast, "Recruiting, Reinvented: How Companies Are Using Social Media In The Hiring Process," Forbes, May 21, 2012. Retrieved from https://www.forbes.com/sites/adp/2016/10/24/how-to-legally-use-socialmedia-to-recruit/\#b0cb2ce29f43

[3] SHRM, "SHRM Survey Findings: Using Social Media for Talent Acquisition - Recruitment and Screening," Society for Human Resource Management, January 7, 2016, Retrieved from https://www.shrm.org/

[4] CareerBuilder, "Number of Employers Using Social Media to Screen Candidates at All-Time High, Finds Latest CareerBuilder Study", June 15, 2017, Retrieved from: http://press.careerbuilder.com/2017-06-15Number-of-Employers-Using-Social-Media-to-Screen-Candidates-atAll-Time-High-Finds-Latest-CareerBuilder-Study

[5] L. McFarland, and R. Ployhart, "Social media: A contextual framework to guide research and practice," Journal of Applied Psychology, vol. 100, n. 6, pp. 1653-1677, 2015.

[6] P. Roth, P. Bobko, C. Van Iddekinge, and J. Thatcher, "Social media in employee-selection-related decisions: A research agenda for uncharted territory," Journal of Management, vol. 42, n. 1, pp. 269-298, 2016.

[7] T. Wirtky, S. Laumer, A. Eckhardt, and T. Weitzel, "On the untapped value of e-HRM: A literature review," Communications of the Association for Information Systems, vol. 38, n. 1, 2016.
[8] C. Backman, and A. Hedenus, "Online privacy in job recruitment processes? Boundary work among cybervetting recruiters," New Technology, Work and Employment, vol. 34, n. 2, pp. 157-173, 2019.

[9] S. Black, D. Stone, and A. Johnson, "Use of social networking websites on applicants' privacy," Employee Responsibilities and Rights Journal, vol. 27, n. 2, pp. 115-159, 2015.

[10] D. Jeske, and K. Shultz, "Using social media content for screening in recruitment and selection: pros and cons," Work, employment and society, vol. 30, n. 3, pp. 535-546, 2016.

[11] P. McDonald, P. Thompson, and P. O'Connor, "Profiling employees online: shifting public-private boundaries in organisational life," Human Resource Management Journal, vol. 26, n. 4, pp. 541-556, 2016.

[12] J. Pike, P. Bateman, and B. Butler, "Information from social networking sites: Context collapse and ambiguity in the hiring process," Information Systems Journal, vol. 28, n. 4, pp. 729-758, 2018.

[13] R. Slovensky, and W. Ross, "Should human resource managers use social media to screen job applicants? Managerial and legal issues in the USA," Info, vol. 14, n. 1, pp. 55-69, 2012.

[14] B. Berkelaar, "Different ways new information technologies influence conventional organizational practices and employment relationships: The case of cybervetting for personnel selection," Human Relations, vol. 70, n. 9, pp. 1115-1140, 2017.

[15] R. Caers, and V. Castelyns, "LinkedIn and Facebook in Belgium: The influences and biases of social network sites in recruitment and selection procedures," Social Science Computer Review, vol. 29, n. 4, pp. 437$448,2011$.

[16] K. Henderson, "They posted what? Recruiter use of social media for selection,” Organizational Dynamics, vol. 48, n. 4, pp. 1-6, 2019.

[17] J. Hoek, P. O'Kane, and M. McCracken, "Publishing personal information online: How employers' access, observe and utilise social networking sites within selection procedures," Personnel Review, vol. 45, n. 1, pp. 67-83, 2016.

[18] N. Melão and J. Reis, "Selecting talent using social networks: a mixedmethods study”, Heliyon, vol. 6, n. 4, p. e03723, 2020. 